\title{
Motor and visual codes interact to facilitate visuospatial memory performance
}

\author{
Marvin Chum \\ University of Toronto, Toronto, Ontario, Canada \\ HAROLD BEKKERING \\ University of Nijmegen, Nijmegen, The Netherlands \\ Michael D. Dodd \\ University of British Columbia, Vancouver, British Columbia, Canada \\ AND \\ JAY Pratt \\ University of Toronto, Toronto, Ontario, Canada
}

\begin{abstract}
The spatial working memory system constantly updates spatial representations and many studies have focused on the underlying principles of the encoding and maintenance of visual information. Here we investigated the question of how the production of actions influences spatial working memory. Participants were given a task that required concurrent maintenance of two spatial arrays, one encoded by visual observation accompanied with pointing movements, the other by only visual observation. Across two experiments, movement during encoding was found to facilitate recognition of spatial arrays in a load-dependent manner. The results suggest an action-based encoding principle within the working memory system, and possible underlying action-related mechanisms are discussed.
\end{abstract}

The fovea, the portion of the retina from which we extract detailed visual information, subtends only a small portion of our visual field. The small size of the fovea is the reason humans make over 150,000 saccadic eye movements each waking day - the information we glean at each fixation is so limited that we must constantly move our eyes from object to object in order to keep an updated spatial representation of our environment. The building and maintenance of this constantly updated spatial representation is the purview of the spatial working memory system.

Most studies examining the operation of the spatial working memory system have focused on the encoding and maintenance of visual information. Typical tasks involve the recall of some aspect of a spatial array which was first presented then removed from view (see, e.g., Luck \& Vogel, 1997). In these tasks, perception plays the important role of regulating the information that reaches our spatial working memory system. Ultimately though, a major role of perception is to provide information to the various actions systems. In other words, perception underlies action (see, e.g., Schneider, 1995; for some recent experimental evidence, see Hannus, Cornelissen, Lindemann, \& Bekkering, 2005).

The reverse can be true as well, in that action can influence perception. The selection-for-action hypothesis (Allport, 1989) proposes that we pay attention only to features in the environment that are relevant toward a certain intended action. For instance, it has been found that people selectively target their first saccadic eye movement in relation to their action intention. If the instruction was to grasp the target, orientation was processed at an enhanced level compared to the condition where observers had to point at the target (Bekkering \& Neggers, 2002; Hannus et al., 2005). Within the realm of spatial cognition, it has been suggested that preparing pointing movements toward a specific cue or object results in our perceiving that object in a more spatially oriented perceptual framework (Fischer \& Hoellen, 2004).

It is evident, therefore, that the relationship between perception, action, and the intervening cognitive processes are quite complex, even within the confines of spatial working memory. Thus, to investigate the seemingly simple question of how pointing influences spatial working memory requires an understanding of how perception and action interact to influence the formation and maintenance of spatial representations; something that is not at all that simple.

It is worth noting that the few studies that have examined the effect of various actions on working memory have found that intervening actions tend to disrupt memory performance. For example, Hale, Myerson, Rhee, Weiss, and Abrams (1996) and Lawrence, Myerson, Oonk, and Abrams (2001), reported that actions produced during 
the retention phase of a spatial working memory task decreased memory performance. The disruptive influence of these intervening (as they occurred between encoding and recall) actions occurred for limb movements alone (Lawrence et al.), saccadic movements alone (Hale et al.), and conjoined limb and eye movements (Hale et al.). Thus, there is good evidence that actions can interfere with working memory processes; the current study looks to examine the other side of the coin and determine if actions can also facilitate spatial working memory performance.

Unlike the intervening task methodologies used in the aforementioned studies, the present study employs a novel paradigm to examine the possible interaction between perception and action in spatial working memory. Specifically, the purpose of the present study is to determine whether encoding spatial information by concurrent perceptual coding and motor action produces better spatial working memory performance than when information is encoded solely in perceptual code. To determine whether motor activity can aid spatial performance, we have developed a task that requires concurrent maintenance of two spatial arrays, one of which will be encoded only through passive visual observation (the no-move array), whereas the other will be encoded by visual observation accompanied with limb pointing movements (the move array). During encoding, the sample arrays will be presented one item at a time on a computer touch-screen display. Upon testing, only one array (move or no-move) will be presented, which may be identical to the sample array, or may have one item shifted in position. Recognition performance will be measured by a same-different judgment.

\section{EXPERIMENT 1}

\section{Method}

Participants. Twenty-five University of Toronto undergraduate psychology students participated in the experiment in exchange for course credit. All participants had either normal or corrected-to-normal vision, submitted informed consent prior to undergoing the experiment, and were naive to its purpose. One participant's data were removed from the study because of a failure to comply with the instructions.

Equipment. The experiment was conducted in a dimly lit soundattenuated testing room, on a PC computer with a 21-in. ELO touch screen monitor at a resolution of $1,024 \times 768$ pixels. The monitor was angled up at $47^{\circ}$ toward the participants, who were instructed to seat themselves in front of screen so that they could comfortably point on the touch screen.

Procedure. The basic sequence of events is depicted in Figure 1. Each trial started when the participant tapped a designated start target on the touch screen located on the vertical midline near the bottom of the monitor. In the study phase of each trial, two spatial arrays were presented one after the other. Both arrays contained the same number of items (varying between three, four, and five items) on every trial. The two arrays differed only in the shape of the items presented: white-filled circles for one array, and white-filled squares for the other array (both subtending $1^{\circ}$ of visual angle). The items in each array were presented sequentially, and their locations were randomized with the constraint that no item occurred in a location previously occupied by another item. Each item disappeared either after $1,000 \mathrm{msec}$ or after the participant tapped on it, whichever occurred first. There were 32 trials for each array size, for a total of 96 trials.

On each trial, one of the arrays constituted the no-move array while the other array constituted the move array. In the no-move array, participants were instructed to simply watch the sequentially presented items and attempt to remember their spatial locations. In the move array, participants were instructed to remember the spatial locations of the items while at the same time tapping, on the touch screen, at the location of each item during the time it was presented.

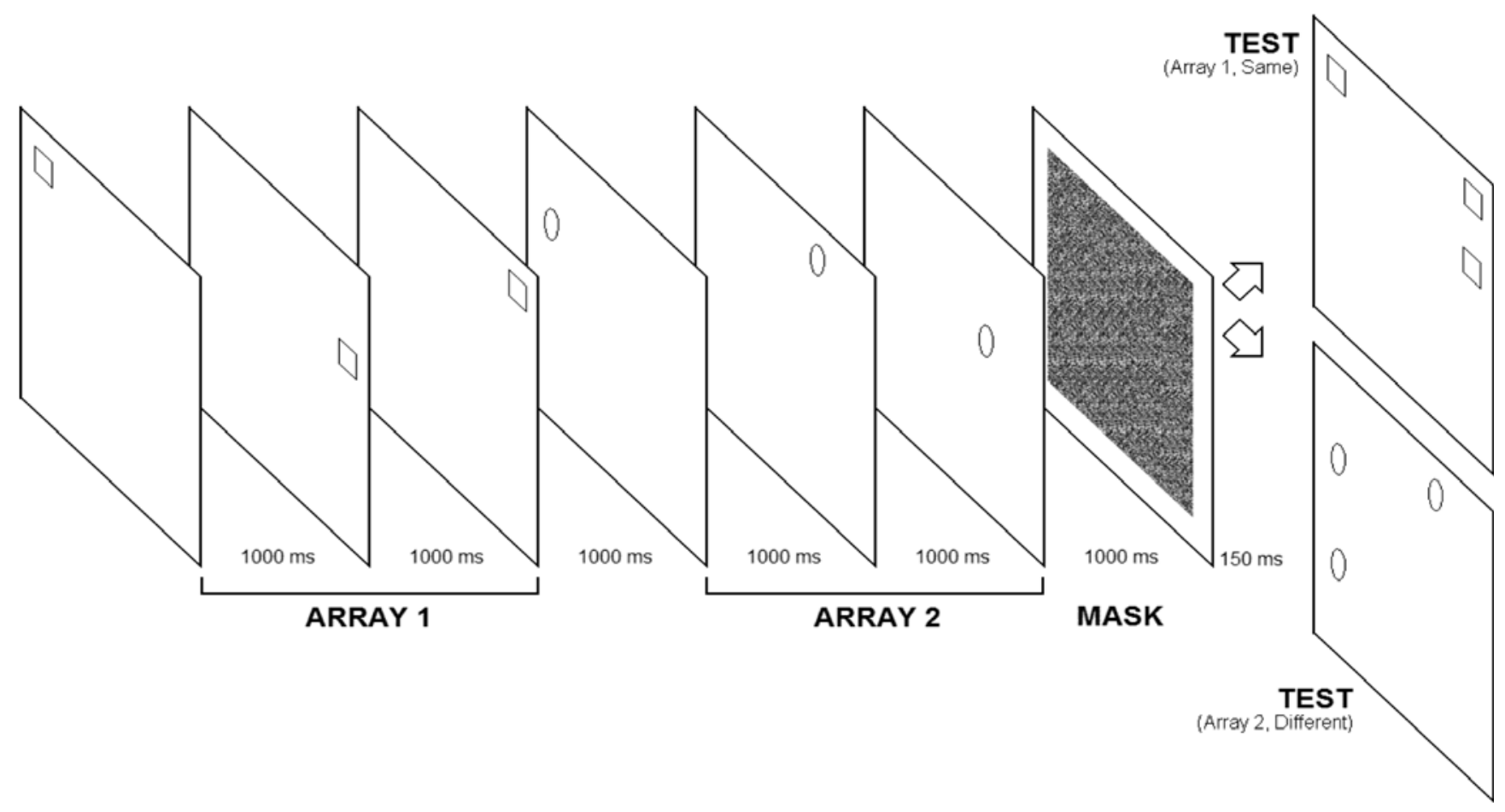

Figure 1. Depiction of a single trial within the experiment, with an array size of three items each, the second array being tested, and the correct response being different. Some participants were instructed to tap the squares, and others were instructed to tap the circles. 
Thus, the no-move array was encoded via a perceptual code while the move array was encoded with both a perceptual and a motor code. Half of the trials for each participant presented the no-move array first, followed by the move array, and the opposite was true for the other half of the trials. The order of these trials was randomly determined for each participant. The different stimuli (circles or squares) were used on the different array types to help the participants distinguish which task was which (e.g., for one group of participants, the no-move array consisted of sequentially presented circles while the move array consisted of sequentially presented squares).

The test phase occurred directly after the study phase. First, a mask containing a random matrix of small black and white squares (with each square subtending $0.4^{\circ}$ of visual angle and the entire mask subtending $12^{\circ}$ ) was presented for $150 \mathrm{msec}$. Following the mask, a test screen appeared containing all of the items from one of the arrays (i.e., either circles or squares). Participants had to judge whether the items presented in the test screen matched what had been seen during the encoding phase for that particular array. Responses of same or different were given by tapping one of two circles on the bottom of the screen, one on the left side that contained an "S" for same, and one on the right side containing a "D" for different. On half of the trials, the test screen reproduced the items as presented during the encoding phase while on the other half of the trials one item was shifted to a previously unoccupied (in both conditions) position. The participants were encouraged to respond within a 5,000-msec time limit, but to focus on response accuracy over time. At the end of the experiment, each participant was asked what strategies, if any, were used to complete the task.

\section{Results}

Recognition accuracy as a function of array order (whether the study array that the test array was based on was presented first or second during study), array size, and pointing instruction can be found on Figure 2. To determine whether spatial working memory was influenced by whether individuals made pointing limb movements during array presentation, recognition accuracy was analyzed with a 2 (array order: first or second) $\times 2$ (pointing instruction: move or no move) $\times 3$ (array size: three, four, or five) ANOVA. Typical memory effects were found, with main effects for array size $[F(2,46), p<.05$; accuracy decreased as array size increased] and array order $[F(1,23), p<.05$; better accuracy when tested on arrays presented second in the study phase]. Importantly, however, the analysis also revealed a main effect for encoding $[F(1,23), p<.05]$, with higher recognition performance when tested on move arrays. This main effect was modulated by the interaction between encoding and array size $[F(2,46), p<.05]$, since the advantage for the recognizing move arrays decreased with increasing array size. No other interactions reached significance $\left(F_{\mathrm{S}}<1\right)$.

\section{EXPERIMENT 2}

One possible confound in the first experiment was that the move condition might have induced people to more actively process the item locations, and this deeper encoding resulted in the better performance at the low loads rather than any action processes, per se. To examine this possibility, Experiment 2 was a replication of Experiment 1, with the exception that participants were required to verbally report the location of the items in the no-move condition.

\section{Method}

Participants. Thirteen University of Toronto undergraduate psychology students participated in the experiment in exchange for course credit. All participants had either normal or corrected-tonormal vision, submitted informed consent prior to undergoing the experiment, and were naive to its purpose. None had participated in the previous experiment.

Equipment and Procedure. The equipment was exactly the same as used previously. The only changes in procedure were (1) only three-item arrays were used ( 32 trials total), since these showed the largest effect in Experiment 1, and (2) participants verbally reported whether each item in the no-move condition was to the left or right of the vertical midline of the display (they were instructed to guess on items falling directly on the midline), and (3) the items in both the move and no-move conditions were displayed for $1,000 \mathrm{msec}$.

\section{Results}

Recognition accuracy, as a function of array order and pointing instruction, can be seen in Figure 3. A 2 (array order: first or second) $\times 2$ (pointing instruction: move or no move) ANOVA showed main effects for array order $[F(1,12)=$ $46.0, p<.001]$ and pointing instruction $[F(1,12)=9.9$, $p<.01]$, replicating the findings from Experiment 1 . No interaction effect was found $[F(1,12)<1]$. In the no-move condition, for the nonmidline items, participants accurately reported the location of $97.1 \%$ of the items.

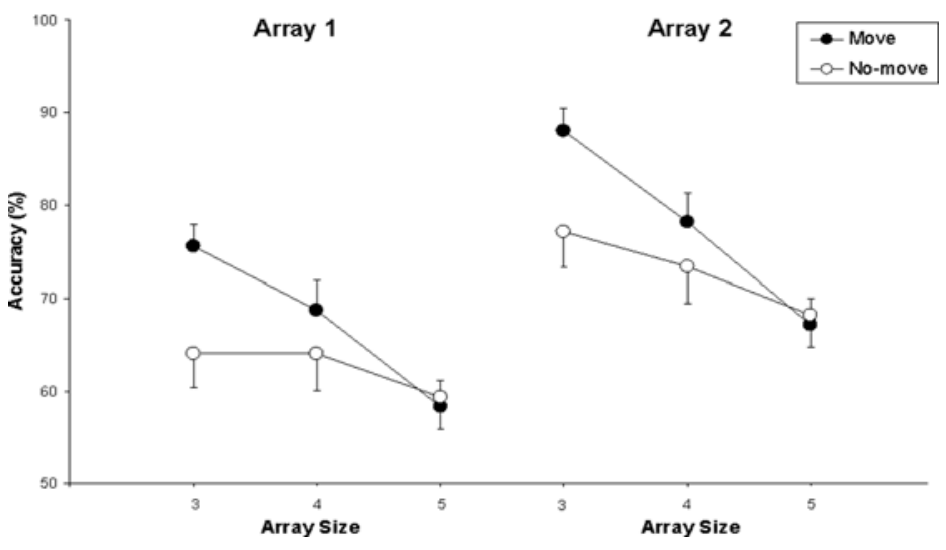

Figure 2. Recall accuracy at various array sizes, divided into the arrays tested (first and second), for Experiment 1. Error bars depict 95\% confidence intervals. 


\section{DISCUSSION}

In the present experiment, we examined how action and perception interact to influence performance on a spatial working memory task. This was done using a paradigm requiring that participants maintain two spatial arrays simultaneously, one of which was encoded visually and by action (move array), whereas the other was encoded only by visual inspection (no-move array). We found that recognition accuracy was higher for move arrays than for no-move arrays. Furthermore, this facilitation in memory performance was found to be load-dependent; when the memory load was lowest, the facilitation from motor action was more profound, but at the highest load the facilitation had very little effect. In addition, two typical memory effects were found. One was that recognition accuracy for the first studied array was lower than for the second studied array, a typical effect of temporal proximity between the studied information and the test phase. The other was that increasing the number of to-be-remembered items resulted in decreased recognition performance for both arrays, as expected because of increased task difficulty.

The improved spatial working memory performance for the move arrays is consistent with the results of previous studies investigating the effects of finger pointing on visual perception. Indeed, there is general agreement that pointing encourages a spatial-based perceptual framework more so than simply viewing objects in the visual field. This can be seen in Fischer and Hoellen (2004), who found that participants who were instructed to point to the targets had identical RTs for invalid locations, regardless of whether the locations were on cued or uncued objects (object-based differences did occur with button-lifting movements). Likewise, Linnell, Humphreys, McIntyre, Laitinen, and Wing (2005), using a similar paradigm, reported that preprogrammed pointing led to impaired discrimination of invalid cued-object targets, even when pointing was conducted in a manner that forced the participant to first perceive the placeholders as being part of an object. These studies provide evidence that pointing

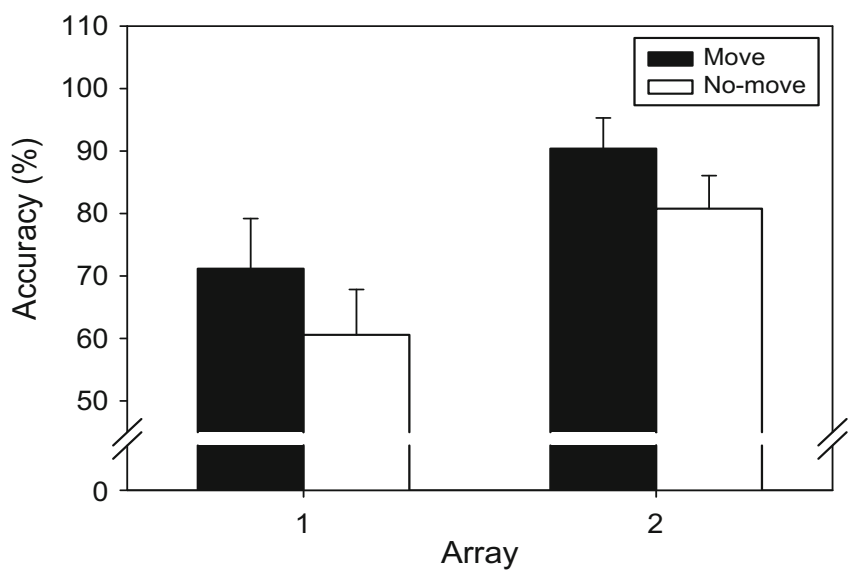

Figure 3. Recall accuracy, divided into the arrays tested (first and second), for Experiment 2. Error bars depict $95 \%$ confidence intervals. leads attention to emphasize space-based representations, a conclusion consistent with the present findings.

As noted earlier, the notion that action may facilitate the generation of spatially based frames of reference is consistent with the selection-for-action hypothesis (Allport, 1987, 1993), which posits that information from the environment is selectively attended on the basis of its relevance to an intended action. If pointing promotes a spatial-based perceptual framework, then pointing might improve the salience of the relevant features of the spatial arrays - that is, the spatial arrangement. This would result in improved encoding of the arrays, explaining the improved recognition performance on arrays that were actively encoded.

The facilitating effects of action-based encoding may also be related to the enactment effect, which refers to the improvement in recall of action word phrases when encoding is accompanied by the performance of action (Mohr, Engelkamp, \& Zimmer, 1989; Saltz \& Donnenwerth-Nolan, 1981; for a review, see Engelkamp, 1998). Much of the literature (e.g., Hornstein \& Mulligan, 2001; Zimmer \& Engelkamp, 1985) explains the facilitation in terms of allocentric and egocentric information. For example, Zimmer and Engelkamp distinguished between kinematic (spatial location in visual space) and kinesthetic (muscle feedback) information components, and reported a partial dissociation in their representation in memory. Furthermore, Hornstein and Mulligan ruled out motor imagery as the critical factor behind the enactment effect by showing that memory of items encoded actively is significantly better than memory for items encoded by passive observation of another person's action. So it seems that the enactment effect is highly rooted to cognitive processes activated only with self-performed actions.

In the present experiment, action-based encoding may have involved the differential encoding of two types of spatial information: egocentric (body-based) and allocentric (scene-based). By making pointing movements to the presented items, participants may have engaged in a stronger form of egocentric encoding than when simply viewing the items. This is because allocentric encoding is considered a function of the hippocampus and the parietal cortex (see, e.g., DiMattia \& Kesner, 1988; Kesner, Farnsworth, \& DiMattia, 1989; Save \& Moghaddam, 1996), and should have occurred in both no-move and move conditions. Egocentric coding, however, involves the striatum of the basal ganglia (De Leonibus, Lafenetre, Oliverio, \& Mele, 2003; De Leonibus, Oliverio, \& Mele, 2005) and would be more likely to occur when limb movements are planned and produced. The egocentric code generated in the move condition would be integrated and retained with allocentric information in the parietal cortex, as proposed by both Save and Moghaddam (1996) and de Bruin, Moita, de Brabander, and Joosten (2001). It is possible, therefore, that action-based encoding enhanced recognition performance through both increased spatial-based perceptual selection and increased egocentric coding.

The finding of a load-dependent decrease in the actionbased recognition facilitation also bears discussion. The decrease may be due to the fact that allocentric spatial strategies have been proposed to be preferentially applied 
over egocentric spatial strategies (Save \& Moghaddam, 1996). As a result, any facilitation offered by egocentric strategies may be masked or even negated in more complex situations, such as with a larger array size. An alternative explanation would be that large arrays simply exceed the capacity available for storing egocentrically coded spatial information in spatial working memory. It is known that the maximum capacity for spatial working memory lies between four and five items (Luck \& Vogel, 1997), corresponding to the array size at which there is least facilitation. Hence, the larger the array size, the smaller the facilitation by proportion, leading to a convergence in recognition accuracy. It is also possible that there is a trade-off relationship between motor action and spatial working memory rehearsal. With larger arrays, the demands required to execute the pointing movements may interfere with the increasing attentional resources required to rehearse a larger spatial array. This possibility is consistent with the conclusions drawn by Awh, Jonides, and Reuter-Lorenz (1998), who suggested that shifts of attention away from a spatial target during rehearsal would impact subsequent memory for that target's location. At the moment, it is difficult to ascertain which of these accounts, or which combinations, might have yielded the load-dependent decrease in action-based facilitation.

In conclusion, the present experiment shows that when two spatial arrays - one actively encoded, the other passively encoded - are kept in mind simultaneously, the actively encoded array stands a better chance to be retrieved with higher accuracy. We propose that the advantage is caused by either increased spatial-based perceptual bias caused by the act of pointing, or because of the formation of a stronger egocentric spatial map that works in parallel with an allocentric map, or some integration of the two factors. Finally, this facilitation was found to be dependent on the size of the spatial array, in that facilitation decreased with a larger array. This load-dependent effect was postulated to be due to a natural bias in favor of allocentric code, a limitation of egocentric spatial memory capacity, or a trade-off relationship between the resources required for rehearsal versus action planning and execution. Although more studies are needed to pinpoint the exact underlying cognitive mechanisms of the interactions presented, clearly, the present study recommends further incorporation of action concepts in the field of working memory.

\section{AUTHOR NOTE}

M.D.D. is now at the University of Nebraska, Lincoln. This research was supported by a grant from the Natural Sciences and Engineering Research Council of Canada to J.P. Please address correspondence to J. Pratt, Department of Psychology, University of Toronto, 100 St. George Street, Toronto, ON, M5S 3G3 Canada (e-mail: pratt@psych.utoronto.ca).

\section{REFERENCES}

AlLPORT, A. (1987). Selection for action: Some behavioral and neurophysiological considerations of attention and action. In H. Heuer \& A. F. Sanders (Eds.), Perspectives on perception and action (pp. 395419). Hillsdale, NJ: Erlbaum.
Allport, A. (1989). Visual attention. In M. I. Posner (Ed.), Foundations of cognitive sciences (pp. 631-782). Cambridge, MA: MIT Press.

Allport, A. (1993). Attention and control: Have we been asking the wrong questions? A critical review of twenty-five years. In D. E. Meyer \& S. Kornblum (Eds.), Attention and performance XIV (pp. 183-218). Cambridge, MA: MIT Press.

Awh, E., Jonides, J., \& Reuter-Lorenz, P. A. (1998). Rehearsal in spatial working memory. Journal of Experimental Psychology: Human Perception \& Performance, 24, 780-790.

BeKKering, H., \& NegGers, F. S. W. (2002). Visual search is modulated by action intentions. Psychological Science, 13, 370-374.

de Bruin, J. P. C., Moita, M. P., De Brabander, H. M., \& Joosten, R. N. J. (2201). Place and response learning of rats in a Morris water maze: Differential effects of fimbria fornix and medial prefrontal cortex lesions. Neurobiology of Learning \& Memory, 75, 164-178.

De Leonibus, E., Lafenetre, P., Oliverio, A., \& Mele, A. (2003). Pharmacological evidence of the role of dorsal striatum in spatial memory consolidation in mice. Behavioral Neuroscience, 117, 685-694.

De Leonibus, E., Oliverio, A., \& Mele, A. (2005). A study on the role of the dorsal striatum and the nucleus accumbens in allocentric and egocentric spatial memory consolidation. Learning \& Memory, 12, 491-503.

DiMattia, B. V., \& Kesner, R. P. (1988). Spatial cognitive maps: Differential role of parietal cortex and hippocampal formation. Behavioral Neuroscience, 102, 471-480.

EngelKamp, J. (1998). Memory for actions. Hove, U.K.: Psychology Press/Taylor \& Francis.

Fischer, M. H., \& Hoellen, N. (2004). Space- and object-based attention depend on motor intention. Journal of General Psychology, 131, 365-377.

Hale, S., Myerson, J., Rhee, S. H., Weiss, C. S., \& Abrams, R. A. (1996). Selective interference with the maintenance of location information in working memory. Neuropsychology, 10, 228-240.

Hannus, A., Cornelissen, F. W., Lindemann, O., \& Bekkering, H. (2005). Selection-for-action in visual search. Acta Psychologica, 118, 171-179.

Hornstein, S. L., \& Mulligan, N. W. (2001). Memory of action events: The role of objects in memory of self- and other-performed tasks. American Journal of Psychology, 114, 199-217.

Kesner, R. P., Farnsworth, G., \& DiMattia, B. V. (1989). Double dissociation of egocentric and allocentric space following medial prefrontal and parietal cortex lesions in the rat. Behavioral Neuroscience, 103, 956-961.

Lawrence, B. M., Myerson, J., Oonk, H. M., \& Abrams, R. A. (2001). The effects of eye and limb movements on working memory. Memory, 9, 433-444.

Linnell, K. J., Humphreys, G. W., McIntyre, D. B., Laitinen, S., \& WiNG, A. M. (2005). Action modulates object-based selection. Vision Research, 45, 2268-2286.

LUCK, S. J., \& Vogel, E. K. (1997). The capacity of visual working memory for features and conjunctions. Nature, 390, 279-281.

Mohr, G., EngelKamp, J., \& Zimmer, H. D. (1989). Recall and recognition of self-performed acts. Psychological Research, 51, 181-187.

Saltz, E., \& DonnenwerTh-Nolan, S. (1981). Does motoric imagery facilitate memory for sentences? A selective interference test. Journal of Verbal Learning \& Verbal Behavior, 20, 322-332.

Save, E., \& Moghaddam, M. (1996). Effects of lesions of the associative parietal cortex on the acquisition and use of spatial memory in egocentric and allocentric navigation tasks in the rat. Behavioral neuroscience, 110, 74-85.

SCHNEIDER, W. X. (1995). VAM: A neuro-cogitive model for visual attention control of segmentation, object recognition, and space-based motor action. Visual Cognition, 2, 331-376.

Zimmer, H. D., \& ENGELKamp, J. (1985). An attempt to distinguish between kinematic and motor memory components. Acta Psychologica, $\mathbf{5 8}, 81-106$.

(Manuscript received August 28, 2006; revision accepted for publication January 9, 2007.) 